\title{
Féeries
}

Études sur le conte merveilleux, XVII $-\mathrm{XIX}{ }^{\mathrm{e}}$ siècle

\section{"J'ai enchâssé des vers en beaucoup d'endroits. » Poésie et prose dans la Psyché de La Fontaine}

"I Have Inserted Verses in Many Places." Poetry and Prose in La Fontaine's

Psyche

\section{Benjamin Bouchard}

\section{OpenEdition}

\section{Journals}

Édition électronique

URL : https://journals.openedition.org/feeries/1038

DOI : 10.4000/feeries.1038

ISSN : 1957-7753

Éditeur

UGA Éditions/Université Grenoble Alpes

Édition imprimée

ISBN : 978-2-37747-012-9

ISSN : 1766-2842

\section{Référence électronique}

Benjamin Bouchard, « " J'ai enchâssé des vers en beaucoup d'endroits. » Poésie et prose dans la Psyché de La Fontaine », Féeries [En ligne], 14 | 2017, mis en ligne le 31 juillet 2017, consulté le 21 septembre 2021. URL : http://journals.openedition.org/feeries/1038 ; DOl : https://doi.org/10.4000/ feeries. 1038

Ce document a été généré automatiquement le 21 septembre 2021.

(c) Féeries 


\title{
" J'ai enchâssé des vers en beaucoup d'endroits. » Poésie et prose dans la Psyché de La Fontaine
}

\author{
"I Have Inserted Verses in Many Places." Poetry and Prose in La Fontaine's \\ Psyche
}

Benjamin Bouchard

Contes et nouvelles en vers en 1665 et 1666, Fables choisies mises en vers en 1668 : avant de publier en 1669 Les Amours de Psyché et de Cupidon, La Fontaine s'est employé à donner une forme poétique à ses originaux (Ésope, Boccace) ou à la leur conserver (Phèdre, l'Arioste) ; et le travail de versification lui a paru assez important pour être signalé dans les titres. Au rebours, il présente tout d'abord Psyché comme "une fable contée en prose $^{1}$ ", choix d'autant plus surprenant que, dit-il, les " aventures [de ses héros], étant pleines de merveilleux en beaucoup d'endroits, [lui] demandaient quelque chose d'héroïque et de relevé ». Dans la langue littéraire du xvII siècle, le mot d'hérö̈que évoque immanquablement le poème héroïque, « où l'on fait la description de quelque action, ou entreprise extraordinaire ", écrit Furetière. D'où une première question : pourquoi avoir choisi la prose pour Psyché? La Fontaine avait apparemment de quoi justifier qu'il mît en vers le récit d'Apulée. Puis, après s'être déterminé pour la prose, pourquoi a-t-il "enchâssé des vers en beaucoup d'endroits » (p. 126) ? Pour qu'on « trouvât, nous dit-il, du solide » dans son œuvre (ibid.) : la raison est brève et n'est pas très claire. Enfin, ce mélange de prose et de vers ne s'accorde guère, semble-t-il, avec le souci affiché dans la «Préface » de se conformer à «la règle la plus étroite que nous ayons », celle de «l'uniformité de style »; car si d'un côté l'histoire de Psyché semble réclamer différents "caractères » («simple», «orné », "galant », « héroïque et [...] relevé »), d'un autre côté « il n'est pas permis [d']employer l'un en un endroit, et l'autre en un autre ». Or voici que, trois pages plus loin, le même auteur nous annonce sans sourciller qu'il a mêlé des vers à la prose! Difficile de concilier cette bigarrure manifeste avec l'uniformité de style. La Fontaine dit avoir cherché un «juste 
tempérament »; ce mot nous entraîne du côté de la musique ; comment le transposer au domaine de la littérature?

\section{«Nos vers lassent à la longue. »}

2 L'idée de prose poétique et de poème en prose n'est pas inconnue du $\mathrm{XVII}^{\mathrm{e}}$ siècle. À l'époque, la versification peut être une condition suffisante mais non nécessaire pour parler de poésie. À cet égard il faut noter que, selon Furetière, le mot poème ne se dit que « fort abusivement » des genres lyriques, comme le sonnet, et que « les vrais Poëmes sont les Epiques \& les Dramatiques $»^{2}$. Ces derniers, en effet, nécessitent d'inventer une fable, ce qui est pour les néo-aristotéliciens le propre du poète - c'est la poésie comme fiction. La poésie peut encore se définir comme «la langue des dieux» (p.3), c'est-àdire comme un discours «le plus fleuri, le plus susceptible d'ornements et de ces figures nobles et hardies qui font une langue à part » (ibid.) - c'est la poésie comme fonction poétique du langage : le vers et la rime sont des ornements importants, mais pas indispensables ${ }^{3}$.

3 Or que La Fontaine conçoive son « conte » (p. 123) de Psyché comme un poème, cela ne fait guère de doute. Deux éléments plaident en ce sens. Tout d'abord l'histoire, qui est pour l'essentiel en prose, se voit dotée d'une sorte de cadre métrique très remarquable. Poliphile prélude à son récit par un « exorde » (p. 134) en vers; il ponctue le passage de la première à la deuxième partie à l'aide d'un quatrain (p.174); enfin il ne «cr[oit] pouvoir mieux finir que par l'hymne de la Volupté » (p. 258). Si le vers et la rime ne sont pas indispensables à la poésie, ils ont bien sûr un statut particulier : la présence de ces seuils versifiés facilite pour le lecteur l'identification de Psyché comme un poème. En outre Les Amours de Psyché et de Cupidon ne sont pas publiés seuls en 1669 : ils forment le premier volet d'un diptyque dont Adonis constitue l'autre panneau. L'« Avertissement » qui précède «ce poème » (p. 3) éclaire rétrospectivement le conte. La Fontaine présente Adonis, qui est tout entier en vers, comme une idylle héroïque. Ce genre, perdu de vue par les traditionnelles histoires de la littérature, était bien vivace dans les esprits du temps ${ }^{4}$. Idylle héroïque traduit l'italien idillio favoloso, c'est-à-dire plus exactement «idylle mythologique». Les aventures amoureuses de la Fable fournissent le sujet de ces poèmes d'inspiration ovidienne, qui font quelques centaines ou quelques milliers de vers ; toutefois Giambattista Marino, qui est l'inventeur du genre, l'a poussé jusqu'aux dimensions gigantesques de son Adone aux 41000 hendécasyllabes. L'histoire de Psyché et de Cupidon est d'ailleurs enchâssée dans l'Adone, où l'on trouve d'autres petits idilli favolosi. Après avoir donc qualifié d'idylle hérö̈que son Adonis, La Fontaine poursuit : « [...] il m’a semblé à propos de ne le point séparer de Psyché.» (p. 799, n. 4) Les deux œuvres sont donc solidaires et participent du même genre. Mais si Psyché est un poème et plus précisément une idylle héroïque, comme Adonis, pourquoi avoir choisi, au rebours d'Adonis, de la conter en prose ? La Fontaine n'explique point cette différence, mais la brièveté de l'un et la longueur de l'autre la justifient implicitement.

4 En effet, dans la Première partie des Contes, notre auteur affirme que "comme les narrations en vers sont très malaisées, il faut se charger de circonstances le moins qu'on peut $^{5}$ ». Or la matière de Psyché est trop riche pour supporter les gênes de la versification. Dans les Contes, La Fontaine alterne deux formules. Son premier mouvement le porte aux vers irréguliers, justement parce qu'ils ont, dit-il, « un air qui tient beaucoup de la prose ${ }^{6}$ ». D'autres récits, plus nombreux, sont en « vieux langage », 
ce qui implique notamment l'emploi de l'ancien «vers commun » qu'est le décasyllabe. Mais la plupart des pièces écrites dans ce mètre-là ne font guère plus de deux cents vers, et aucune n'excède quatre cents. Au-delà de cette limite La Fontaine emploie les vers irréguliers, comme s'il fallait retrouver alors quelques-unes des libertés du sermo solutus. Encore la taille de ces nouvelles en vers libres (cinq à six cents vers) est-elle sans commune mesure avec celle de Psyché. Il semble bien que, passé une certaine longueur, La Fontaine juge les contraintes métriques insupportables au lecteur. En cela il rejoint nombre de ses contemporains. Parlant des narrations, le père Rapin juge en effet que «la monotonie de notre vers alexandrin, peut devenir ennuyeuse dans les pièces de longue haleine ${ }^{7}$ ». Il avait échangé à ce sujet avec Bussy-Rabutin, qui lui écrit : «Et pour répondre à ce que vous me demandez, je conviens qu'un poème épique ne peut réussir en notre langue [...]. Pour les raisons, je n'en sais point d'autres que celles que vous me mandez. Il est vrai que la cadence, les rimes et le repos de nos vers lassent à la longue : ce qui n'arrive pas dans les latins qui plaisent par la diversité8.» L'air de prose et la variété inséparables des vers irréguliers permettent de pallier un temps le défaut d'une métrique absolument régulière ${ }^{9}-$ mais un temps seulement. Pour traiter Psyché de cette manière, il eût fallu tailler dans le vif, et tel n'est évidemment pas le dessein de La Fontaine, puisque loin de retrancher au conte d'Apulée, il ajoute des épisodes et des circonstances, et joint même à l'aventure principale une promenade de Versailles ainsi qu'un petit dialogue socratique sur le rire et la compassion. Il avait fait l'inverse pour Adonis, réduisant l'histoire du malheureux chasseur à une ligne claire, au point de supprimer la jalousie du dieu Mars : économie frappante, surtout comparée au colossal Adone de Marino. L'Adonis, qui compte à peine plus de six cents alexandrins, a justement la longueur maximale des récits en vers que l'on trouve chez La Fontaine: nouvelle confirmation que pour lui la poésie devient fastidieuse au-delà de ce seuil.

Le choix de la prose pour Psyché est donc la conséquence du défaut ou, si l'on veut, du génie du vers français qui, «lass[ant] à la longue », ne peut convenir à une œuvre de longue haleine. Pourtant La Fontaine ne renonce pas tout à fait à la poésie, puisqu'il " enchâss[e] des vers en beaucoup d'endroits »: pourquoi métamorphoser ainsi son poème en prose en prosimètre?

\section{"Sans que de tant d'objets les beautés se confondent. »}

6 La Fontaine donne une raison sibylline à cela ; ce sont parmi les dernières lignes de la « Préface » :

[Je] ne prétends pas que mon ouvrage soit accompli : j'ai tâché seulement de faire en sorte qu'il plût, et que même on y trouvât du solide aussi bien que de l'agréable. C'est pour cela que j'ai enchâssé des vers en beaucoup d'endroits, et quelques autres enrichissements, comme le voyage des quatre amis, leur dialogue touchant la compassion et le rire, la description des enfers, celle d'une partie de Versailles. (p. 126)

Notons d'abord que si la poésie tient du solide, elle ne se confond point avec lui : une partie du voyage et la totalité du dialogue sont en prose. Pour paraphraser le maître de Philosophie : tout ce qui est en vers est solide, mais tout ce qui est solide n'est pas en vers. Par ailleurs, l'adjectif solide s'éclaire de son opposition avec agréable. Pour comprendre ces termes, et donc la fonction de la poésie dans Psyché, il faut se référer à des passages parallèles. La «Préface» du premier recueil des Contes oppose les 
《 ouvrages vraiment solides » aux " galanteries $»^{10}$, c'est-à-dire aux œuvres amusantes et plaisantes sans plus. Le Songe de Vaux distingue de même le "sérieux » du " galant " (p. 97), partage qui se superpose, si curieux que cela nous paraisse, avec celui entre « héroïque » et « lyrique » (p. 78). Solide dans la « Préface » de Psyché ne peut donc que renvoyer à ce qui est nommé plus haut dans le même texte justement l'« héroïque et [le] relevé» (p. 123), tandis qu'agréable reprend les termes de "galant», de " plaisanterie » et de «badineries » (p. 123-124). Le vocabulaire critique de La Fontaine est souple, mais l'on perçoit bien l'opposition entre deux séries de termes : d'un côté ce qui est solide, sérieux, héroïque et relevé, de l'autre l'agréable, le galant, le lyrique, les badineries et les plaisanteries. Le champ de l'écriture de Psyché se situe donc entre deux pôles : disons, pour simplifier, le solide et le galant. La distinction entre prose en poésie est secondaire et ne se superpose que partiellement à la précédente. L'on pourrait donc schématiser ce que dit la "Préface » par un tableau à double entrée : ici la prose et la poésie, là le solide et le galant; une case reste vide: le lecteur peut déduire de la « Préface » qu'il n'y aura point de vers galants dans cette histoire.

Cette analyse du paratexte est confirmée par la lecture de l'œuvre. Parmi les vingt-huit pièces de vers que compte Psyché, deux grands ensembles se détachent. Le premier et le plus nombreux est formé de quatorze descriptions où la poésie est appelée par la présence de la merveille, c'est-à-dire du merveilleux ou bien de l'émerveillement ${ }^{11}$; du reste l'admiration et le surnaturel s'entraînent souvent l'un l'autre: le faste mythologique de Versailles éblouit ses visiteurs, et lorsque c'est l'étonnement seul qui commande d'abord le passage au vers, la Fable intervient toujours à titre d'ornement.

Dans un deuxième groupe de neuf poèmes, le passage au vers parait commandé par l'émotion, mais une émotion qui a toujours un caractère paroxystique : une passion forte, un "transport", dans la langue de l'époque: colère, jalousie, désespoir, mélancolie amoureuse, indignation, enthousiasme poétique ${ }^{12}$. Les états d'âme plus modérés, comme la tendresse, la douceur ou le simple chagrin n'ont pas leur place ici. $\mathrm{Du}$ reste, l'admiration qui accompagne les descriptions merveilleuses du premier groupe est une forme de "ravissement", pour user d'un autre mot du français classique.

Restent cinq fragments. Deux sont des citations poétiques (il s'agit de chansons ${ }^{13}$ ), ce qui justifie à soi seul le recours aux vers. Les trois autres peuvent passer pour des exceptions, qui confirment d'autant plus aisément la règle que je ne prétends point que La Fontaine se soit précisément formulé une règle pour l'emploi des vers dans son ouvrage $^{14}$.

11 Descriptions merveilleuses et transports ont à voir avec l'extraordinaire : à situation exceptionnelle, langage hors du commun, c'est-à-dire poésie, au double sens de discours versifié et de "langage des dieux», profus en ornements: bref, poésie " héroïque » et « relevé[e] », voudrait-on ajouter, car il semble que ce soient les termes désignant dans la « Préface » le pôle « solide » de l'œuvre lorsqu'il s'agit de vers. Mais il y a là un problème : à lire de plus près les fragments en vers, ces mots d'héroïque et de relevé ne s'appliquent pas également bien à tous. Voilà qui nous impose d'affiner la description.

12 Tout d'abord, il faut supposer que dans l'opposition galant/solide, seul le premier terme est marqué, comme disent les linguistes. Il me semble donc que, dans la «Préface ", La Fontaine considère comme solide tout ce qui n'est pas précisément du galant, de la plaisanterie, de la badinerie. Ensuite il importe de voir que La Fontaine expose par 
étapes les principes qui doivent guider la lecture de Psyché: la «Préface » n'indique que les bornes extrêmes d'un continuum dont les degrés intermédiaires nous sont nommés uniquement lorsque nous franchissons l'ultime seuil de l'œuvre, à savoir l'incipit. Ce dernier présente les quatre amis. Cette petite «Académie» (p.127) est une fiction métapoétique. Chacune de ces figures incarne une couleur stylistique : interprétation rendue évidente par l'étymologie de chaque nom, ainsi que par la polysémie au XVII siècle du mot de caractère, qui désigne à la fois le «tempérament d'une personne » et la "qualité d'un style ». Or La Fontaine joue explicitement sur ce double sens :

[Acante] aimait extrêmement les jardins, les fleurs, les ombrages. Poliphile lui ressemblait en cela; mais on peut dire que celui-ci aimait toutes choses. Ces passions, qui leur remplissaient le cœur d'une certaine tendresse, se répandaient jusqu'en leurs écrits, et en formaient le principal caractère. Ils penchaient tous deux vers le lyrique, avec cette différence qu'Acante avait quelque chose de plus touchant, Poliphile de plus fleuri. Des deux autres amis, que j'appellerai Ariste et Gélaste, le premier était sérieux sans être incommode, l'autre était fort gai. (p. 127-128)

13 Il est donc possible de croiser la première typologie que nous avons proposée avec une autre, fondée sur ces caractères. En effet toutes les pièces de vers peuvent être en quelque sorte attribuées soit à Ariste, soit à Acante, ou à Poliphile, Gélaste étant exclu puisqu'il est du côté du plaisant et que ce registre, dans Psyché, exclut l'usage du vers. La Fontaine invente ses personnages pour que nous percevions bien les inflexions diverses du "langage des dieux", celles-là même qu'il nomme on ne peut plus clairement: il y a des pièces héroïques et relevées, d'autres lyriques et touchantes, d'autres enfin lyriques et fleuries ${ }^{15}$.

Est-ce un hasard si La Fontaine associe les trois premiers fragments en vers de son récit respectivement aux noms d'Acante, d'Ariste et de Poliphile? «Acante [...] ne se put tenir de réciter certains couplets de poésie que les autres se souvinrent d'avoir vus dans un ouvrage de sa façon. » (p. 128) Suivent des stances dont voici la deuxième :

Jasmins dont un air doux s'exhale,

Fleurs que les vents n'ont pu ternir,

Aminte en blancheur vous égale,

Et vous m'en faites souvenir. (p. 129)

15 Vient ensuite la description de la grotte de Téthys; or l'idée de la promenade au château revient à Ariste, lequel «dit qu'il y avait de nouveaux embellissements à Versailles : il fallait les aller voir » (p. 128). Ce sont les premiers vers :

Dans l'un [des bas-reliefs], le dieu du jour achève sa carrière.

Le sculpteur a marqué ces longs traits de lumière,

Ces rayons dont l'éclat, dans les airs s'épanchant,

Peint d'un si riche émail les portes du couchant.

On voit aux deux côtés le peuple d'Amathonte

Préparer le chemin sur des dauphins qu'il monte :

Chaque Amour à l'envi semble se réjouir

De l'approche du dieu dont Téthys va jouir. (p. 130)

Enfin, nous l'avons déjà dit, Poliphile débute l'histoire de Psyché par un exorde en vers :

Le dieu qu'on nomme Amour n'est pas exempt d'aimer :

À son flambeau quelquefois il se brûle ;

$E t$, si ses traits ont eu la force d'entamer

Les cœurs de Pluton et d'Hercule,

Il n'est pas inconvénient

Qu'étant aveugle, étourdi, téméraire, 
Il se blesse en les maniant ;

Je n'y vois rien qui ne se puisse faire [...]. (p. 133-134)

Grâce au trois premières pièces de vers, qui sont des échantillons des trois styles poétiques de Psyché, le lecteur a donc l'occasion de se faire l'oreille. Bien sûr il peut hésiter à associer tel passage poétique à un personnage plutôt qu'à un autre. Toutefois l'important n'est pas de ranger les fragments poétiques dans la bonne case, mais de prendre conscience que si la poésie relève toujours du solide, ce style a bien des nuances, figurées dans les caractères d'Ariste, d'Acante et de Poliphile. Le tableau que j'esquissais tout à l'heure pourrait donc se développer ainsi :

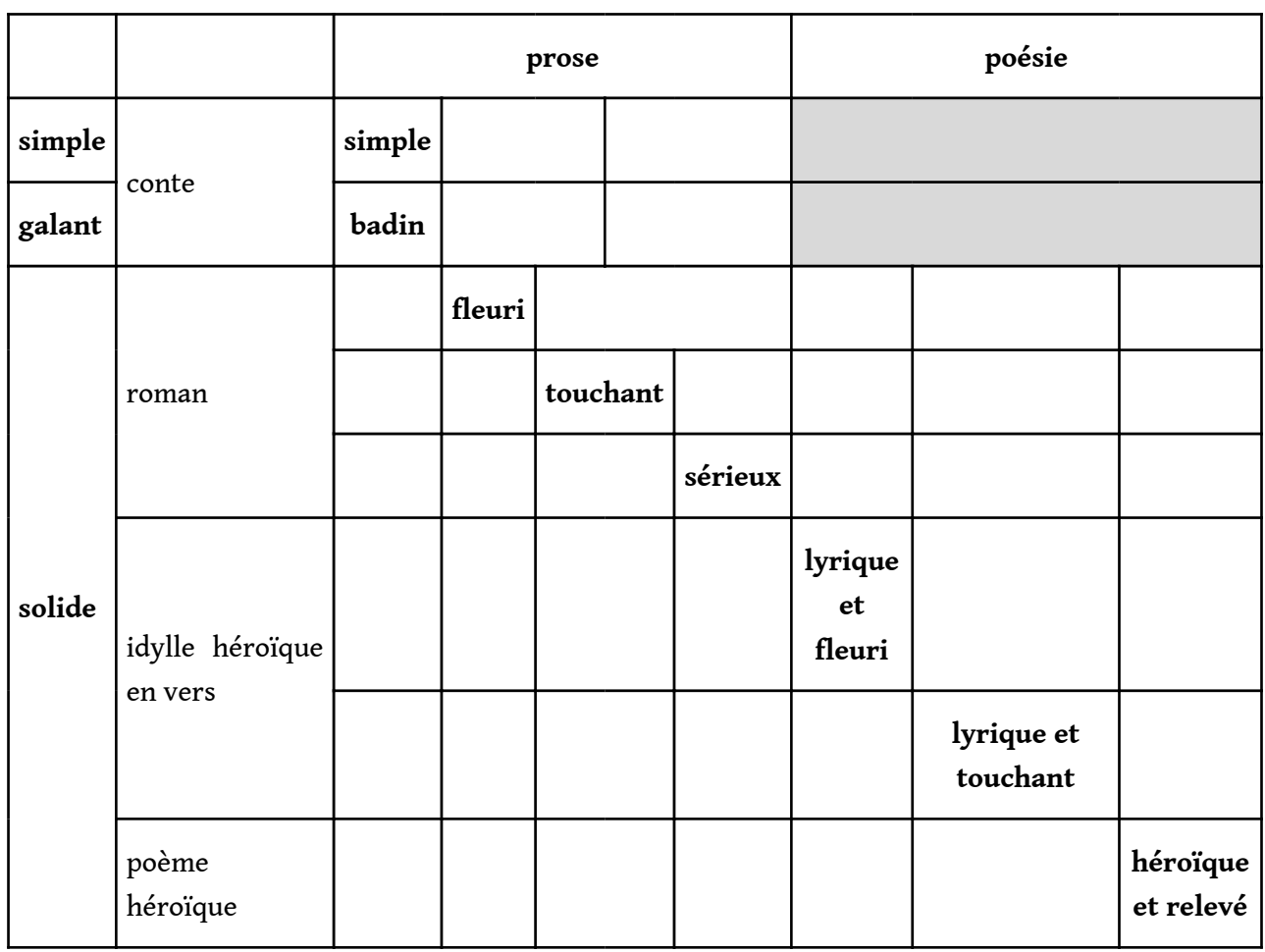

Pour tâcher de rendre exhaustif cette espèce d'échelle des styles dans Psyché, j'y ai intégré la première observation que fait La Fontaine sur le "genre d'écrire qu'[il] a choisi» (p.124): «Je ne savais quel caractère choisir: celui de l'histoire est trop simple ; celui du roman n'est pas encore assez orné ; et celui du poème l'est plus qu'il ne faut. » (p. 123) Le mot d'histoire ne saurait désigner ici l'historiographie; il renvoie à la narratio telle que la présentent les traités de rhétorique savants et scolaires, comme devant avoir un style simple, pur et clair. Si La Fontaine mentionne ce style, c'est parce que, vue sous un certain angle, l'histoire de Psyché est, comme disaient les Anciens et Apulée avec eux, un "conte de vieille", un "conte de ma mère l'Oye", selon l'expression reçue bien avant Perrault. Quand La Fontaine écrit Psyché, les contes de fées n'ont pas de statut littéraire ; ce merveilleux-là n'a pas les titres de noblesse de la Fable : « [...] un conte comme celui-ci [...] est plein de merveilleux, à la vérité, mais d'un merveilleux [...] propre à amuser les enfants [...].»(p.123) En vertu du principe rhétorique de l'aptum, le seul ornement de ce genre est de n'en avoir aucun; mais l'histoire de Psyché appartient aussi à la Fable : ce statut insaisissable (Psyché sera tour à tour, dans ses aventures, princesse, bergère, esclave puis déesse) - ce statut, dis-je, séduit La Fontaine pour ses potentialités stylistiques. 
19 Pour revenir à notre question, en enchâssant des vers dans la prose, La Fontaine enrichit considérablement la variété de Psyché, et l'on sait combien cela est important pour lui. Cependant il ne faut pas céder au déterminisme de l'œuvre achevée: La Fontaine aurait pu traiter en prose les passages qui sont en vers. Que serait-il arrivé ? Y aurait-il eu seulement cinq couleurs stylistiques dans Psyché : la prose simple, badine, fleurie, touchante et sérieuse? - Non pas : le «langage des dieux » appelle naturellement le vers et la rime, mais il peut s'en passer, comme en témoignent les «Vers en prose » de Pellisson ${ }^{16}$. La disparition des vers aurait donc atténué, non pas gommé, les différences entre les styles. Pour le dire autrement, la versification accentue la diversité, elle permet de rendre la variété des tons plus vive et plus remarquable, "sans que de tant d'objets les beautés se confondent» (p.187) - ou risquent de le faire aux yeux du lecteur distrait... Non seulement, donc, l'ambitus de la poésie dans Psyché admet plusieurs nuances, mais le relief des vers en général sur la surface de la prose apporte au lecteur le plaisir d'une diversité plus intense.

Cependant l'on ne peut qu'être frappé de la contradiction, au moins apparente, entre d'une part ce choix formel, qui accroît l'hétérogénéité des parties de l'œuvre, et d'autre part le désir affiché de respecter «la règle [...] étroite» de «l'uniformité de style». Pour se conformer à cette règle, La Fontaine déclare: "J'avais donc besoin d'un caractère nouveau, et qui fût mêlé de tous ceux-là ; il me le fallait réduire dans un juste tempérament. » (p.123) Force est de constater, à lecture de Psyché, que la pratique contredit la théorie: La Fontaine se permet bel et bien d'employer les vers en un endroit et la prose en un autre. À l'évidence, son dessein n'est pas de mêler plusieurs caractères de façon à obtenir une pâte homogène, comme le peintre qui, amalgamant avec soin deux couleurs primaires, en obtient une troisième parfaitement unie. Foin du tempérament, donc? ou faut-il le comprendre autrement que comme un style uniforme?

\section{« J'ai cherché ce tempérament avec un grand soin. »}

21 La règle de l'uniformité de style vient de la poétique néo-aristotélicienne. Dans ce système, les genres et les styles doivent être bien séparés les uns des autres; les mêler dans une œuvre, c'est heurter le bon goût comme une dissonance blesse l'oreille. D'où, par exemple, la condamnation du genre de la tragi-comédie. Le père Rapin est tout à fait représentatif de ce néo-aristotélisme: «[...] le Tasse, écrit-il, est fort défectueux, qui mêle dans son poème le caractère badin avec le sérieux, et toute la force et la majesté de la poésie héroïque à la délicatesse de l'églogue et de la poésie lyrique ${ }^{17}$. " Toujours à propos de La Jérusalem délivrée, on lit plus loin: "[...] il y mêle tant de galanterie et d'affectation, qu'il oublie souvent la gravité de son dessein, et la dignité de son caractère. » De même Bouhours : «[...] son génie l'emporte quelquefois trop loin : il est trop fleuri en quelques endroits : il badine dans des rencontres assez sérieuses. »

Les néo-aristotéliciens ont donc une interprétation très stricte de l'unité de ton et leur force institutionnelle est considérable. La Fontaine le sait bien, qui écrit : « D'employer l'un en un endroit et l'autre en un autre, il n'est pas permis. » (p. 123) Cependant notre auteur fait le choix de la liberté grande. Sa position est parfaitement exprimée dans un texte qu'on lui a longtemps attribué, mais qui est en fait de la main de Nicole : «[...] on veut qu[e les tragédies, les comédies, les satires, etc.] aient chacune leur caractère particulier, dont il ne soit pas permis de s'éloigner ; mais malgré toutes ces règles, les 
hommes croiront toujours avoir droit d'être indulgents à ceux qui ne les violeront que pour leur plaire.»(p.781) Selon moi, l'étendard de l'uniformité de style que La Fontaine brandit d'abord dans sa «Préface » n'est qu'un leurre. À preuve, ce tour de magie critique par lequel il escamote la notion d'uniformité au profit de celle de tempérament.

La Fontaine prend la peine dans cette " Préface » à tous égards capitale de préciser qu'il " aime extrêmement la musique ». Il était sans doute musicien - claveciniste - luimême (p. 667). Je ferai l'hypothèse qu'il songe dans cette "Préface » au tempérament musical, comme le voisinage des mots de réduire et juste paraît l'indiquer. En effet, depuis l'époque romantique le tempérament des instruments est égal, c'est-à-dire que tous les intervalles de la gamme sont identiques. Partant, toutes les tonalités ont le même coloris sonore, la seule différence sensible étant entre les modes majeur et mineur. Il en résulterait une grande monotonie pour une oreille baroque. En ce tempslà, le tempérament consistait à définir des intervalles inégaux : certains étaient dits " purs » (parfaitement justes), d'autres étaient réduits, et lorsqu'on les utilisait, ils sonnaient «durs» (disons "faux»). Dans un tempérament inégal chaque tonalité possède une couleur bien marquée, qu'il était courant d'associer à un sentiment ou à un caractère. Dans ses Règles de composition en 1690, Marc-Antoine Charpentier propose ainsi une liste des "Propriétés des modes ", où l'on retrouve quelques-uns des termes mêmes que La Fontaine emploie pour les quatre amis : sérieux, gai, tendre, solitaire et mélancolique, champêtre, etc. Le musicien baroque doit choisir son tempérament, c'est-à-dire donner la priorité à certains intervalles en fonction de la tonalité fondamentale qui est celle de sa pièce. Quand il module, les tonalités éloignées de la fondamentale seront sinon tout à fait impraticables, du moins feront entendre des dissonances, qui peuvent d'ailleurs être recherchées pour leur expressivité, lorsqu'il s'agit par exemple de faire naître chez l'auditeur un sentiment d'angoisse, de tension, ou simplement de créer la surprise.

Ce modèle musical peut aider à comprendre l'écriture de Psyché. En effet, juste après avoir prononcé le mot de tempérament, La Fontaine explique quel sera le ton dominant de son œuvre : « [...] il a fallu badiner depuis le commencement jusqu'à la fin ; il a fallu chercher du galant et de la plaisanterie. Quand il ne l'aurait pas fallu mon inclination m'y portait, et peut-être y suis-je tombé en beaucoup d'endroits contre la raison et la bienséance.» (p.123-124) L'identification de cette tonalité fondamentale lui paraît tellement importante qu'il la rappelle dans la fiction elle-même à la faveur d'un dialogue essentiel entre les quatre amis: " "Quand Poliphile [...] ne ferait pas tant le pathétique, la chose n'en irait que mieux, vu la manière d'écrire qu'il a choisie." Le sentiment de Gélaste fut approuvé. » (p. 175)

De ces lignes il ressort que si Psyché est écrite dans une tonalité fondamentale (la gaieté), le conteur module à certains moments dans d'autres tonalités, c'est-à-dire qu'il emploie, contrairement à ce que déclare la "Préface », un caractère " en un endroit, et l'autre en un autre », ce que j'ai tâché de mettre en évidence dans le tableau proposé plus haut.

À ce point, la question que se posent les musiciens est technique et propre à leur art : comment définir les intervalles de telle sorte que, la tonalité fondamentale étant posée, toutes les modulations envisagées soient possibles? Si l'on veut inventer, à partir du paradigme musical, des instruments d'analyse textuelle, il faut trouver un ou des équivalents stylistiques de l'opération musicale d'altération des intervalles. Celle-ci 
revient à modifier les rapports sonores entre les tonalités, c'est-à-dire encore à modifier l'effet produit par le passage d'une tonalité à une autre: comment, donc, modifier l'effet produit par le passage d'une couleur stylistique à une autre? Il y a quatre façons de le faire :

1. En jouant sur l'intensité de la couleur, plus ou moins vive ;

2. En modifiant la durée pendant laquelle une couleur passe au premier plan avant de s'effacer ;

3. En opérant une transition plus ou moins graduelle d'une couleur à l'autre ;

4. En proposant une motivation plus ou moins forte pour le changement de couleur.

27 Je prendrai rapidement quelques exemples de chacun de ces procédés dans Psyché, en montrant au besoin comment ils s'entraînent l'un l'autre, conjuguent leurs effets, voire se confondent.

Pour comprendre la notion d'intensité, qui repose sur une analogie picturale et non plus musicale, il faut avoir à l'esprit qu'une couleur stylistique résulte de la combinaison de traits textuels de diverses natures (isotopies, figures, rythmes, énonciation). Selon que ces caractéristiques sont en plus ou moins grand nombre, l'effet produit est plus ou moins intense, exactement comme un rouge, un jaune, un bleu peuvent être plus ou moins vifs, pâles ou profonds. La nature des traits influence aussi la perception: Hermogène, dont Les Catégories du style furent si importantes aux $\mathrm{XVI}^{\mathrm{e}}$ et $\mathrm{XVII}^{\mathrm{e}}$ siècles, montre bien que la "pensée " (nos "isotopies ", "motifs » ou "thèmes ») contribue à l'effet stylistique plus fortement qu'aucun autre composant du discours ${ }^{18}$.

$\mathrm{Au} \mathrm{XVII}{ }^{\mathrm{e}}$ siècle le paradigme de la poésie héroïque est la grande manière virgilienne : il est plus hérö̈que de parler de batailles, de dieux et de héros guerriers que d'amour. Or La Fontaine n'enfle jamais sa voix si haut. Il écarte même explicitement une telle intensité héroïque :

Je laisse ces sujets aux maitres du Parnasse ;

Et pendant que Louis, peint en dieu de la Thrace,

Fera bruire en leurs vers tout le sacré vallon,

Je le célébrerai sous le nom d'Apollon. (p. 132)

La majorité au moins des pièces de vers relève bien de ce que La Fontaine nomme le "solide ", mais en abordant des sujets réputés moins nobles (l'amour, le charme des jardins, etc.), La Fontaine atténue la couleur et limite l'amplitude de l'écart avec sa tonalité fondamentale. D'autant plus qu'à l'autre bout du spectre, le plaisant est lui aussi d'une intensité modérée: il ne va jamais jusqu'à la gauloiserie scabreuse de certains passages des Contes. Dans les deux ou trois moments où la sexualité est matière à badinage (p. 136, 143, 214), l'euphémisme le plus élégant est de rigueur, par exemple lorsque, Poliphile ayant loué la majesté du cortège marin de Vénus, Gélaste l'interrompt : " "Cela devait être beau, dit[-il]; mais j'aimerais mieux avoir vu votre déesse au milieu d'un bois, habillée comme elle était quand elle plaida sa cause devant un berger." Chacun sourit de ce qu'avait dit Gélaste. » (p. 136) Ce passage est le plus osé de toute l'œuvre! Une édition de Psyché conservée au British Museum de Londres porte des corrections qui sont probablement de la main de La Fontaine ; les lignes que nous venons de citer sont barrées, et l'on peut lire dans la marge : "Ôter cela dans une réimpression. » (p. 825) Preuve, s'il en est, de la modération extrême que s'impose dans Psyché l'auteur des « Lunettes » ou du « Tableau ». 
31 La préférence pour des couleurs plaisante et sérieuse point trop accusées produit un effet d'ensemble sur toute l'œuvre; cependant le procédé de l'atténuation d'une couleur est parfois d'une portée plus limitée, comme dans les vers où Vénus exprime sa colère. Lues un peu vite et d'un peu loin, ces strophes où le style est noble et parfois rude ne manquent pas de grandeur : la passion qui s'y exprime est forte, les figures sont nombreuses; cela pourrait passer pour des stances hétérométriques dans le haut style de Malherbe ou de Racan. Or, dans une étude sur Psyché, Benoît de Cornulier propose une savante et subtile analyse de ce poème, d'où il ressort que les choix métriques de La Fontaine donnent à ce passage un air un peu vieillot: "Vénus, beauté passée de mode, s'en plaint en un rythme goûté un siècle avant le récit de Poliphile, mais passé de mode et même d'un autre âge ${ }^{19}$. "Bref, derrière la solennité apparente de ce «transport » (Poliphile parle des « extrémités où s'emporta la déesse » [p. 135]), il y a une sorte de persiflage métrique qui diminue très subtilement l'intensité de la couleur sérieuse. On voit au passage comment il est possible de modifier la vivacité d'un effet en superposant différentes couleurs : c'est ainsi, par exemple, que les passages en vers libres, «ayant un air qui tient beaucoup de la prose ", contribuent à leur manière au tempérament ${ }^{20}$.

Il est évident que plus le passage en vers est long, plus la modulation laisse une impression profonde. Les pièces de vers dans Psyché ont des tailles très différentes, du simple distique aux deux ou trois pages d'alexandrins. Cependant, la proportion d'ensemble des vers par rapport à la prose est faible. En outre, il n'y a que deux très amples descriptions dans Psyché; les autres poésies sont plutôt brèves. La Fontaine observe manifestement une grande retenue. Comme souvent chez lui, ce choix formel est souligné sinon explicité. Le conteur écarte ainsi, au début de l'histoire, la possibilité d'un sonnet ou d'un madrigal néo-pétrarquiste : «Je ne m'amuserai point à chercher des comparaisons jusque dans les astres pour vous la représenter assez dignement: c'était quelque chose au-dessus de tout cela, et qui ne se saurait exprimer par les lis, l'ivoire ni le corail. Elle était telle enfin que le meilleur poète aurait de la peine à en faire une pareille.»(p.134) Plus loin, dans l'évocation des merveilles du palais d'Amour, matière à poésie s'il en est, la prose prend le relais des vers à deux reprises, sans doute afin d'éviter une trop longue modulation poétique (p. 146-147).

Les transitions de Psyché sont très souvent d'abord des coutures grammaticales. La Fontaine use de la syntaxe comme d'un fil reliant les deux tissus de la prose et de la poésie. Ici les vers sont intégrés à la phrase (p. 155); là un simple « dit-il » assure la liaison (p.128); presque toujours le conteur fait usage de reprises anaphoriques, comme dans cet extrait :

Ces spectacles se terminaient par le coucher du Soleil.

Il était témoin de la fête,

Paré d'un magnifique atour ;

Et caché le reste du jour,

Sur le soir il montrait sa tête.

Mais comment la montrait-il ? environnée d'un diadème d'or et de pourpre, et avec

toute la magnificence qu'un roi des astres peut étaler. (p. 154 ; je souligne.)

À l'instar de celle-ci, rares sont les pièces de vers que l'on pourrait supprimer sans dommage, c'est-à-dire sans que la cohérence grammaticale et sémantique du texte en pâtisse. La transition syntaxique agit souvent de concert avec une transition stylistique, c'est-à-dire un effet de dégradé. Dans notre dernier exemple, la prose file superbement la métaphore royale: c'est le même «langage des dieux» que dans le quatrain, la 
versification en moins. L'on ne passe donc pas abruptement de la poésie héroïque à la prose badine, mais le changement de régime se fait en douceur.

La présence de vers dans la prose peut être plus ou moins motivée. On retrouve ici la notion cardinale de la poétique classique, à savoir la vraisemblance, sous les deux espèces de la bienséance interne et externe. Il faut en outre distinguer deux cas de figures, qui sont deux manières différentes de servir le tempérament.

Premièrement la poésie est le fait d'un personnage qui compose, cite ou chante des vers : il faut que cette attitude soit conforme à son caractère ou à la passion qu'il éprouve à ce moment-là (c'est la bienséance interne), mais aussi conforme aux convenances (c'est la bienséance externe). Tous ces éléments justifient l'insertion des stances d'Acante sur l'Orangerie :

La beauté et le nombre des orangers et des autres plantes qu'on y conserve ne se sauraient exprimer. Il y a tel de ces arbres qui a résisté aux attaques de cent hivers. Acante, ne voyant personne autour de lui, ne se put tenir de réciter certains couplets de poésie que les autres se souvinrent d'avoir vus dans un ouvrage de sa façon. (p. 128)

Il est naturel de trouver des vers ici, puisqu'un personnage en récite ; il agit ainsi parce que cela est non seulement conforme à son ethos de poète lyrique et tendre porté au champêtre tel qu'il vient d'être décrit au lecteur, mais encore au pathos d'admiration qui le saisit alors ("[il] ne se put tenir»); cependant un poète, même transporté d'admiration, ne déclame pas ses ouvrages à la première occasion, à moins d'être fou : voilà pourquoi le narrateur précise que les conditions d'intimité lui permettant de le faire de façon bienséante sont réunies. On voit avec quel soin La Fontaine motive la première apparition de la poésie dans sa prose.

Second cas de figure : le passage aux vers est le fait du narrateur. La vraisemblance ne consiste plus seulement en une conformité à la rhétorique des caractères et des passions : la justification interne de la poésie sera un autre principe rhétorique, celui de la convenance stylistique (aptum). D'où ce thème, répété comme un leitmotiv : la prose, cette "langue naturelle de tous les hommes » (p.123) ne peut exprimer ce qui dépasse de trop loin l'expérience commune. Par exemple :

La beauté et le nombre des orangers et des autres plantes qu'on y conserve ne se sauraient exprimer. (p. 128)

Ceci est proprement matière de poésie : il ne siérait guère bien à la prose de décrire une cavalcate [sic] de dieux marins : d'ailleurs je ne pense pas qu'on pût exprimer avec le langage ordinaire ce que la déesse parut alors :

C'est pourquoi nous dirons en langage rimé etc. (p. 136)

De représenter à quel point l'affliction se trouva montée, c'est ce qui surpasse mes forces :

L'éloquence elle-même, impuissante à le dire,

Confesse que ceci n'est point de son empire etc. (p. 141)

Il existe, dans l'Antiquité comme à la Renaissance, deux manières différentes et presque opposées de concevoir l'aptum : soit il sert à déterminer le ton idoine pour l'ensemble de l'œuvre, étant donné son sujet principal (de là vient la règle de l'uniformité de style); soit les auteurs considèrent que la question du style approprié se pose pour chaque nouvel élément du discours ou du récit (de là vient l'esthétique de la diversité). La Fontaine adopte bien sûr la conception d'un aptum exacerbé ${ }^{21}$, et ce dès la "Préface»: «Mes personnages me demandaient quelque chose de galant, leur 
aventures étant pleines de merveilleux en beaucoup d'endroits, me demandaient quelque chose d'héroïque et de relevé. » (p. 123)

Ici encore la modulation stylistique peut posséder en outre une bienséance externe : le changement de tonalité doit être attendu par un lecteur que la notion même de bienséance externe suppose peu ou prou néo-aristotélicien. Or c'est le genre de l'œuvre qui détermine l'horizon d'attente: il n'est pas bienséant que le tragédien introduise une scène plus légère pour relâcher quelque peu la tension, que le poète épique parle d'amour comme le poète lyrique, ou que l'auteur comique suscite, fût-ce brièvement, la crainte et la pitié. Nous avons suggéré de voir en Psyché une idylle héroïque ; toutefois, pour des raisons trop longues à déduire, cette filiation est à peine avouée par La Fontaine et ne peut nous être utile ici. Pour l'étude du tempérament, il est plus intéressant de voir notre auteur faire participer son livre d'un autre genre ou, si l'on veut, d'une autre veine: Psyché est une œuvre galante, et ce n'est pas un hasard si la "Préface », lieu du pacte de lecture et de la mise en place de l'horizon d'attente, insiste beaucoup là-dessus. En associant le galant, la plaisanterie et la badinerie, La Fontaine se place sous le patronage de Vincent Voiture. Ce prince des poètes galants, oublié aujourd'hui mais dont tout le XVII siècle célébra le génie, avait, dans les années 1630-1640, remis au goût du jour la poésie de Marot et des rhétoriqueurs ${ }^{22}$. D'anciennes formes devenues nouvelles par la grâce de la mode trouvèrent ainsi leur place en marge des grands genres de la poétique néo-aristotélicienne. Le prosimètre fait partie de ces formes ressuscitées. Jean-François Sarasin compose, à l'occasion de la mort de son aîné, une éblouissante Pompe funèbre de Voiture, virtuose exercice de style qui non seulement mêle la prose et les vers, mais offre une sorte d'anthologie de tous les genres littéraires goûtés et pratiqués par Voiture ${ }^{23}$. On voit quel intérêt pour La Fontaine à situer Psyché dans le courant de la galanterie : dans ce cadre, le prosimètre, inconcevable ou plutôt inconvenant partout ailleurs, devient acceptable, même pour le néo-aristotélicien - à condition qu'il ne soit pas trop snob ou grincheux et ne veuille rien connaître au-delà de son Aristote et de son Horace.

Il existait dans les salons du XvII ${ }^{e}$ siècle un divertissement nommé le Jeu des Pensées ${ }^{24}$. Une personne choisit une " pensée » et, sans la révéler à ses partenaires, demande à chacun d'associer cet objet inconnu à un autre, de lui faire un "présent » et de le « loger » : une fois le secret révélé, chaque joueur doit improviser de justes raisons aux rapprochements qu'il a opérés. Sur une pensée galante (les yeux de la princesse de Conti), le duc de La Rochefoucauld propose: une partie d'échecs, un basilic, une pyramide égyptienne; le comte de Bassompierre: un loup, un soupir, l'arc-en-ciel. L'histoire ne dit pas ce qu'ils improvisèrent ensuite... Simplifions le jeu et supposons la «pensée » connue : il s'agit, on s'en doute, de Psyché. Je proposerai, comme présent, une anémone, comme logis, un jardin de Le Nôtre, et comme association, un clavecin.

L'anémone est la fleur née du sang d'Adonis. Le poème consacré par La Fontaine à l'amant de Vénus est publié avec Psyché, et ils gagnent à être lus ensemble : tous deux sont des idylles héroïques, mais l'un est un chef-d'œuvre de classicisme ou, comme on disait alors, d' " atticisme "; l'autre, ample, riche, complexe, profus en développements et en ornements est une œuvre de trop d'étendue pour une versification française que La Fontaine juge, comme ses contemporains, fastidieuse à la longue.

La lecture de Psyché, qui se déroule d'une certaine manière dans le parc de Versailles, y ressemble. Depuis les terrasses, le regard du promeneur file sur les longues perspectives aériennes du jardin à la française : tout est fluide, simple et clair; cela 
coule de source quoique ce soit éminemment difficile à concevoir, comme la prose de La Fontaine. Puis on entre dans la futaie et l'on découvre les éblouissants bosquets baroques, si différents par ailleurs les uns des autres. Les enchâssements de vers dans la prose de Psyché font de l'œuvre un espace où chaque « endroit » offre une expérience esthétique singulière et d'autant plus vive qu'elle est assez nettement isolée des autres. Diversité des expériences esthétiques, mais surtout séparation de celles-ci dans le temps et l'espace :

[...] ne vaut-il pas mieux, dis-moi ce qu'il t'en semble,

Qu'on ne puisse sentir tous les plaisirs ensemble,

Et que, pour en goûter les douceurs purement,

Il faille les avoir chacun séparément?

( «À M. de Niert. Sur l'Opéra », p. 616)

Le clavecin est un instrument dont le tempérament est inégal : selon le genre de pièce que l'on joue, il faut réduire certains intervalles entre les notes; cela rend impraticable certaines tonalités, mais surtout cela dote chacune d'une couleur sonore bien spécifique : gai, tendre, touchant, sérieux, etc. La "devise» de La Fontaine, c'est "diversitée ${ }^{25}$ » : il ne veut pas d'un style uni; en revanche, il s'emploie à unifier la prose et les vers par quatre procédés, qui alternent, se conjuguent ou se confondent : jeu sur l'intensité de la couleur stylistique, sur sa durée, sur la transition entre les couleurs (purement grammaticale ou par dégradé), enfin sur leur motivation par une vraisemblance aussi bien interne qu'externe.

Le succès des Contes et des Fables fut immense du vivant de La Fontaine. Psyché fut un échec. Le tempérament choisi n'a-t-il pas été jugé assez «juste »? L'œuvre était-elle trop déliée, trop subtile? La Fontaine acceptait ce risque: "J'ai cherché ce tempérament avec un grand soin : que je l'ai ou non rencontré, c'est ce que le public m'apprendra. » (p. 123) Il parle ici de ses contemporains, mais il ajoute à la dernière ligne de la «Préface»: "[...] quelque peu d'assurance qu'ait un auteur qu'il entretiendra un jour la postérité, il doit toujours se la proposer autant qu'il lui est possible, et essayer de faire les choses pour son usage. » (p. 126)

\section{NOTES}

1. J. de La Fontaine, Euvres complètes, t. II, Euvres diverses, P. Clarac (éd.), Gallimard, coll. "Bibliothèque de la Pléiade ", 1958, p. 123 pour cette citation et les suivantes dans l'introduction, sauf indication contraire. Afin de réduire le nombre de notes, j'indique désormais dans le corps de l'article les références à cette édition, qui comprend notamment, outre Psyché, Adonis et Le Songe de Vaux.

2. Voir aussi la définition de «Poësie» dans le Dictionnaire de l'Académie françoise, Paris, JeanBaptiste Coignard, 1694: "L'art de bien raconter ou representer en vers les actions et les passions humaines sous des fictions ingenieuses. La Poësie est appelée le langage des Dieux. [...] Poesie, Se prend aussi quelquefois seulement pour l'Art de faire des Vers, pour la simple versification. » 
3. Pour s'en convaincre, il faut lire une œuvre aussi curieuse qu'instructive de P. Pellisson, «Vers en prose, prose en vers", dans A. Viala (dir.), L'Esthétique galante: Discours sur les Euvres de Monsieur Sarasin et autres textes de Paul Pellisson, Toulouse, Société de littératures classiques, coll. « Rééditions des textes du XVII ${ }^{\mathrm{e}}$ siècle », 1989, p. 75-77.

4. B. Bouchard, «Idylles héroïques italiennes et françaises au début du XVII siècle ", dans C. Barbafieri et J.-Y.Vialleton (dir.), Vices de styles et défauts esthétiques (XVII-XVIII ${ }^{e}$ siècle), Paris, Classiques Garnier, à paraître.

5. J. de La Fontaine, CEuvres complètes, t. I, Fables et Contes, J.-P. Collinet (éd.), Gallimard, coll. «Bibliothèque de la Pléiade ", 1991, p. 605.

6. Ibid., p. 651.

7. R. Rapin, S. J., Les Réflexions sur la poétique de ce temps et sur les ouvrages des poètes anciens et modernes, E. T. Dubois (éd.), Genève-Paris, Droz/Minard, 1970, p. 82, chap. X.

8. Ibid., cité p. 168, n. 133.

9. J'entends par là où la périodicité est respectée au niveau du vers et de la strophe : sur cette notion, voir B. de Cornulier, «La Fontaine n'est pas un poète classique : pour l'étude des vers mêlés ", Cahiers du Centre d'études métriques, n 1, mai 1992, p. 15-31. Voir aussi Théorie du vers, Paris, Seuil, coll. «Travaux linguistiques », 1982 et Art poëtique : notions et problèmes de métrique, Lyon, Presses universitaires de Lyon, coll. « IUFM », 1995.

10. J. de La Fontaine, ouvr. cité, p. 555.

11. P. 128, 130, 136, 138, 145, 146, 149, 154, 172, 185, 239, 242, 245 (solidaire du précédent) et 259.

12. Soit respectivement p.135, 141, 156 (Psyché compose ce sonnet dans un accès de " glucomorie, qui lui pervertit tous les sens et la ravit comme à elle-même [p. 155, je souligne] ») et $205 ;$ p. 166 et 231 pour l'indignation; p. 133, 174 et 257 pour l'enthousiasme.

13. P. 143 et 233.

14. Pour résorber ces anomalies, il faudrait prendre en compte le rôle de ponctuation que jouent les passages en vers dans la composition de l'œuvre : cela m'entraînerait trop loin du cœur de cette étude.

15. On voit que dans Psyché, l'adjectif lyrique passe du côté de l'héroïque, alors qu'il y est opposé, nous l'avons vu, dans la "Préface " du Songe de Vaux. Il ne me paraît pas que cela fragilise mon hypothèse. Encore une fois, le vocabulaire critique de La Fontaine est souple et largement déterminé ad hoc. En outre, la description par les paratextes et les seuils des différentes couleurs de l'œuvre est plus fine dans Psyché que dans Le Songe.

16. P. Pellisson, ouvr. cité.

17. R. Rapin, S. J., ouvr. cité, p. 86 ; p. 94 et 155 n. 42 pour les deux suivantes ; même idée p. 83.

18. Une synthèse commode des Catégories du style se trouve dans M. Patillon, Éléments de rhétorique classique, Paris, Nathan, coll. « Nathan-Université », 1990. Le point que je rappelle est mentionné p. 98.

19. «Aspects du papillonnage métrique de La Fontaine dans Les Amours de Psyché et de Cupidon, non sans un petit rappel de Maistre Clement", Cahiers du Centre d'études métriques, $\mathrm{n}^{\circ} 3$, février 1997, p. 83.

20. B. Bouchard, «Un air de prose. Les vers irréguliers chez La Fontaine », Poétique, $\mathrm{n}^{\circ} 170$, avril 2012, p. 195-218.

21. Voir P. Galand-Hallyn et F. Hallyn (dir.), Poétiques de la Renaissance : le modèle italien, le monde franco-bourguignon et leur héritage en France au XvI siècle, Genève, Droz, coll. "Travaux d'humanisme et Renaissance ", 2001, p. 532-558.

22. A. Génetiot, Les Genres lyriques mondains (1630-1660). Étude des poésies de Voiture, Vion d'Alibray, Sarasin et Scarron, Genève, Droz, coll. «Histoire des idées et critique littéraire ", 1990. La galanterie a fait l'objet d'un nombre considérable de travaux : parmi eux, je retiendrai, du même auteur, Poétique du loisir mondain, de Voiture à La Fontaine, Paris, Honoré Champion, coll. « Lumière classique », 1997. 
23. J.-Fr. Sarasin, Euvres, t. I, Poésies, P. Festugière (éd.), Paris, Honoré Champion, 1926.

24. D. Denis, Le Parnasse galant: institution d'une catégorie littéraire au XVII siècle, Paris, Honoré Champion, coll. « Lumière classique », 2001, p. 257-258.

25. J. de La Fontaine, ouvr. cité, p. 863.

\section{RÉSUMÉS}

Lorsque l'on examine les rapports de la poésie avec la prose dans la Psyché de La Fontaine, trois questions se posent :

1. Psyché est, dans les termes du XvII ${ }^{\mathrm{e}}$ siècle, une "idylle héroïque ", c'est-à-dire un épisode amoureux tiré de la Fable. Ce genre appelle en principe le vers. Dès lors, pourquoi choisir de conter cette histoire principalement en prose ? Parce que La Fontaine veut faire de Psyché une œuvre de longue haleine et qu'il pense, comme beaucoup de ses contemporains, que «nos vers lassent à la longue ».

2. Cependant, après s'être déterminé pour la prose, pourquoi La Fontaine a-t-il « enchâssé des vers en beaucoup d'endroits "? Notre auteur choisit le sujet de Psyché parce qu'il peut, grâce à lui, mêler plusieurs couleurs stylistiques dans une même œuvre, mais il veut le faire « sans que de tant d'objets les beautés se confondent ». Le passage de la prose au vers est l'un des moyens lui permettant de distinguer les divers registres de son œuvre.

3. Mais en procédant ainsi, il va à l'encontre de la règle de l'uniformité de style, si importante dans l'esthétique classique. Comment concilier uniformité et diversité ? La Fontaine invente, par analogie avec la musique, une poétique du tempérament, qui consiste à unifier la prose et les vers par quatre procédés : jeu sur l'intensité de la couleur stylistique, sur sa durée, sur la transition entre les couleurs, sur leur motivation par une vraisemblance aussi bien interne qu'externe.

When one examines the relationship between poetry and prose in La Fontaine's Psyche, three questions arise:

1. Psyche is what is called in the 17th century a "mythological idyll", i.e. a love-story that belongs to ancient mythology. This genre is supposed to be in verse. Why then choose to tell Psyche mainly in prose? Because La Fontaine wanted Psyche to be a long, ambitious piece of work and yet he thought, like many of his contemporaries, that "French verse is boring in the long run".

2. Once he had chosen prose, why then decide to "insert verse in many places"? He picked out the subject matter of Psyche because it enabled him to use various stylistic colours in the same story, but he wanted to achieve that effect "without the beauties of so many objects mixing up". The switch from prose to poetry is one of the means the author used to differentiate between the various tones of his work.

3. However, with such a stylistic choice, La Fontaine ran against the rule of stylistic uniformity that is so important according to French Classical aesthetics. How could he reconcile uniformity and diversity? In order to achieve that, he created a poetics based on the originally musical notion of temperament that consists in playing with the intensity of the stylistic colour, with its length, in providing transitions between the colours, eventually in justifying them through both internal and external verisimilitude. 
INDEX

Mots-clés : La Fontaine, Psyché, idylle héroïque, vers et prose, prosimètre, conte poétique en prose, style, esthétique classique, tempérament musical

\section{AUTEUR}

\section{BENJAMIN BOUCHARD}

Université Rennes 2, CELLAM 